\title{
Homogeneous Distribution of Alumina Nanoparticles in the 6061 Aluminum Alloy via Mechanical Alloying
}

Víctor Mercado ${ }^{1}$, Raúl Perez ${ }^{1}$, Juan Carlos Díaz-Guillén ${ }^{2}$, Melvyn Alvarez-Vera ${ }^{3}$, Hugo ArcosGutierrez ${ }^{4}$, Jan Mayen ${ }^{4}$, Antonio Enrique Salas-Reyes ${ }^{6}$, Adriana Gallegos-Melgar ${ }^{1}$, Maricruz HernandezHernandez ${ }^{1}$ and Jorge Acevedo ${ }^{3}$

${ }^{1}$ CONACYT-Corporación Mexicana de Investigación en Materiales, San Luis Potosí, San Luis Potosi, Mexico, ${ }^{2}$ CONACYT-Corporación Mexicana de Investigación en Materiales, Saltillo, Coahuila de Zaragoza, Mexico, ${ }^{3}$ Corporación Mexicana de Investigación en Materiales, Saltillo, Coahuila de Zaragoza, Mexico, ${ }^{4}$ CONACYT-Centro de Tecnologia Avanzada, San Luis Potosi, San Luis Potosi, Mexico, ${ }^{6}$ Departamento de Ingeniería Metalúrgica, Facultad de Química, Ciudad de México, Distrito Federal, Mexico

The efforts to improve more efficient aluminum alloys makes that researchers around the world pay attention on micro- and nano-reinforced elements. In this way, the synthesis of the suggested aluminum alloys is a very important step when considering their production. In recent years, the aluminum matrix composites synthesized by mechanical alloying, and reinforced with alumina nano-particles tends to be one of the materials that suggest to have high conditions of strength, ductility and corrosion resistant [1, 2]. In this context, it is important to mention that one of the main parameters to consider in the addition of reinforcing elements is the reinforcement homogeneous distribution that conferes to the material their excellent mechanical properties [3].

This research work deals with the steps to follow in order to obtain a homogeneous distribution of alumina nanoparticles in the aluminum matrix composites, where images depicted the good results obtained.

At the beginning, aluminum powders were obtained from the machining process of a commercial alloy 6061 in the form of metal chips and mixed with $5.0 \mathrm{wt}$. \% of alumina nanoparticles with an average size of $25 \mathrm{~nm}$. Next, a high-energy ball milling process was carried out during $5 \mathrm{~h}$, using Argon as an inert atmosphere in the milling process. Finally, specimens were cold consolidated and sintered by $3 \mathrm{~h}$ at 800 $\mathrm{K}$ under vacuum conditions. Sintered products were held al $770 \mathrm{~K}$ and hot extruded into a rod of $10 \mathrm{~mm}$ of diameter.

Fig 1. shows SEM (scanning electron microscopy) micrographs of the homogeneous distribution of alumina nanoparticles in the aluminum matrix composite, where nanoparticles appear agglomerated and homogeneously distributed in the specimens, which demonstrates the efficient of mechanical synthesis in the production of aluminum composites through the manipulation of nanometric reinforcements. Additional characterization by energy dispersive spectroscopy (EDS, Fig. 2) analysis of a selected area in the specimen, shows an oxygen signal, which confirms the presence of alumina nanoparticles.

Results of the present work show that with the correct process, it is possible to generate a homogeneous distribution of nanoparticles in the aluminum metal matrix composites that can be offer the best mechanical properties in this type of materials [4]. 


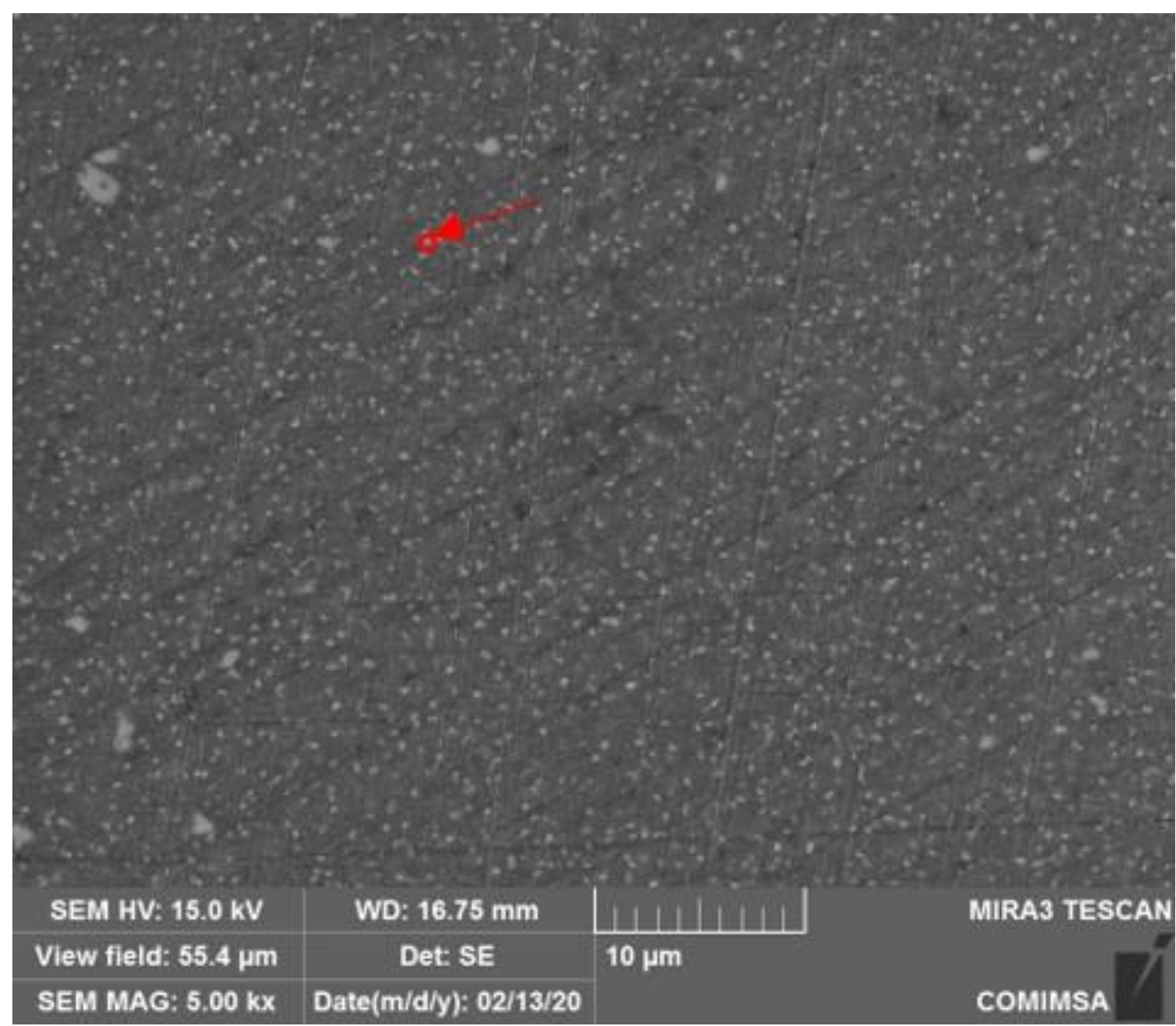

Figure 1. SEM micrograph of the homogeneous distribution of alumina nanoparticles in the aluminum matrix composite 6061, 5000x. 


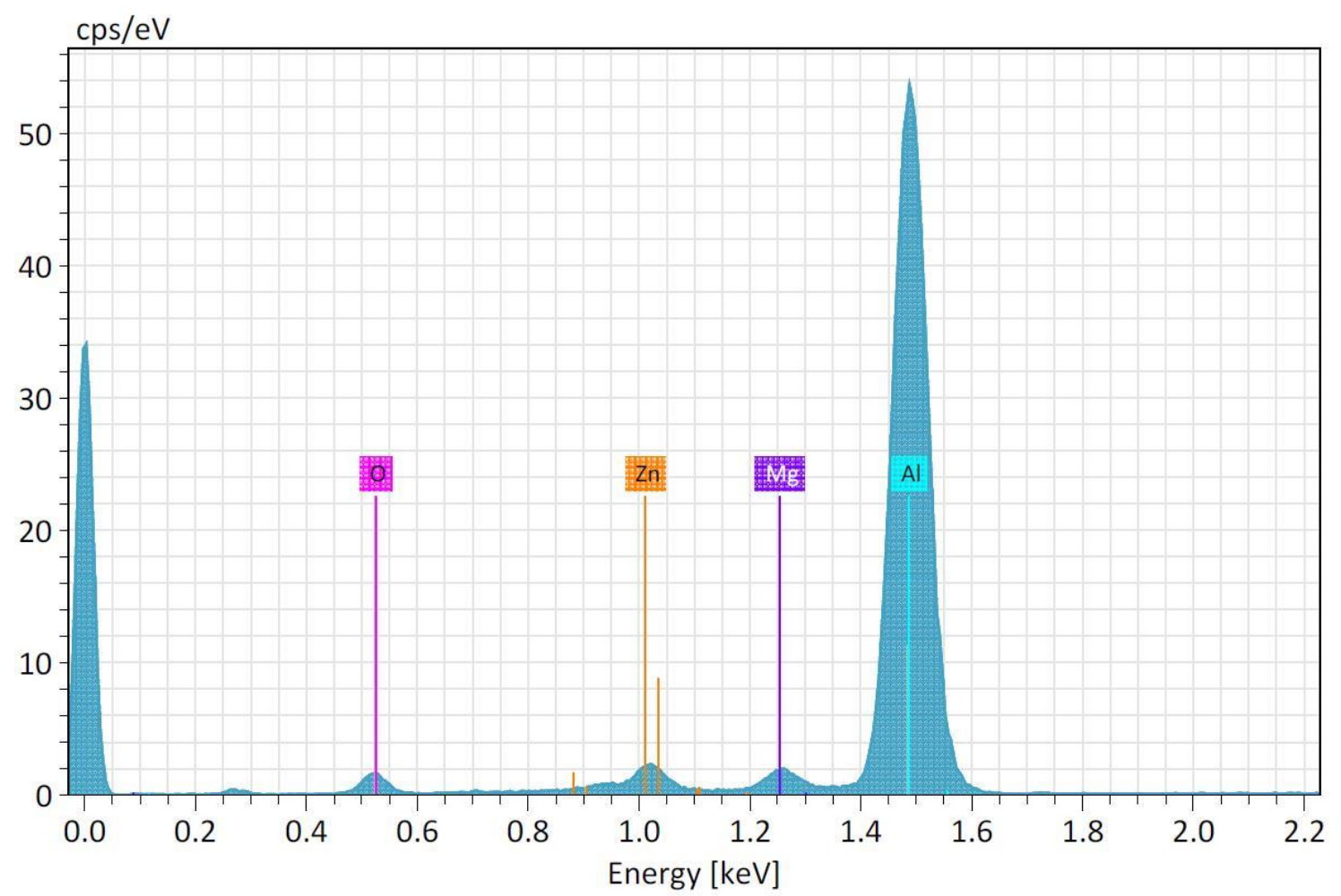

Figure 2. EDS analysis of the selected area in Fig. 1.

\section{References}

[1] PK Krishnan et al, Journal of Alloys and Compounds 784 (2019) p. 1047-1061.

[2] MBN Shaikh et al, Surfaces and Interfaces 15 (2019), p. 166-179.

[3] Z Zahiemyekeh et al, Materials Science and Engineering A 753 (2019), p. 276-284.

[4] The authors are grateful with the program Catedras CONACYT (Project 850 and 674) for their support 